\title{
Gaia: mapping the dynamics and morphologies of Galactic Planetary Nebulae: First results from Gaia Data Release 1
}

\author{
Nicholas A. Walton ${ }^{1}$, Marina Rejkuba ${ }^{2}$, Jeremy R. Walsh ${ }^{2}$ \\ and Albert A. Zijstra ${ }^{3}$ \\ ${ }^{1}$ Institute of Astronomy, University of Cambridge, \\ Madingley Road, Cambridge CB3, OHJ \\ email: naw@ast.cam.ac.uk \\ ${ }^{2}$ European Southern Observatory, \\ Karl-Schwarzschild Strasse 2, 85748, Garching, Germany \\ email: mrejkuba@eso.org and jwalsh@eso.org \\ ${ }^{3}$ Jodrell Bank Centre for Astrophysics, Alan Turing Building, \\ Manchester, M13 9PL, UK \\ email: a.zijlstra@manchester.ac.uk
}

\begin{abstract}
This paper comments on the use Gaia in studying the internal dynamics and morphologies of Planetary Nebulae (PN). It is noted that the second and subsequent releases of Gaia data, will have significant potential in unravelling PN morphologies.
\end{abstract}

Keywords. planetary nebulae, distances, surveys, astrometry.

\section{Introduction}

Planetary Nebulae (PN) are a brief evolutionary stage through which low and intermediate mass stars pass towards the end of their evolution, between red giant and white dwarf. They play an important role in the processing of a number of elements into the surrounding interstellar medium. They can be used as powerful probes of the kinematical structure of the Milky Way, and can provide insights into the chemical evolution history of the Galaxy. Understanding the global role of PN is limited due to large uncertainties in individual distances and to a detailed knowledge of the dynamics of their nebulae. These factors in turn constrain the absolute parameters of PN, such as their sizes, luminosities, masses, lifetimes and determination of the overall Galactic PN population.

We briefly discuss the impact of the ESA Gaia mission data in supporting detailed studies of the expansion histories and morphological evolution of planetary nebulae.

\section{Gaia and the Gaia Data Release 1}

The ESA Gaia satellite was launched at the end of 2013. From the completion of its flight commissioning in July 2014, it collects data over its five year nominal mission to allow the determination of highly accurate positions, parallaxes, and proper motions for $>1$ billion sources brighter than magnitude 20.7 in the white-light photometric band $\mathrm{G}$ of Gaia. Its astrometry is complemented by multi-colour photometry, measured for all sources observed by Gaia, and radial velocities which are collected for stars brighter than $\mathrm{G} \sim 17$. The mission and science goals are summarised in Gaia Collaboration et al. (2016b). 
The first major data release of Gaia data (Gaia DR1) was made 14th September 2016 (Gaia Collaboration et al. 2016a). The Gaia DR1 contains astrometry, G-band photometry, and a small number of variable star light curves, for a total of $\sim 1.14$ billion sources. The two main astrometric components of Gaia DR1 consist of a primary astrometric data set with positions, parallaxes, and mean proper motions for $\sim 2$ million stars in common between the Gaia DR1, Hipparcos and Tycho-2 catalogues and a secondary astrometric data set containing the positions for an additional $\sim 1.14$ billion sources. The primary data set is the realisation of the Tycho-Gaia astrometric solution (TGAS), the principles of this are outlined in Michalik et al. (2015). The typical uncertainty is about 0.3 mas for

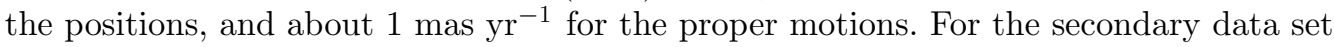
the typical uncertainty on the positions is $\sim 10$ mas. Photometric data is released for all sources in the primary and secondary astrometric data sets, containing the mean Gaia G-band magnitudes. The brightest source in Gaia DR1 has a magnitude G $=3.2$, while the majority of the sources $(99.7 \%$ ) are in the range $11.2 \leqslant \mathrm{G} \leqslant 21$ (van Leeuwen et al. 2016).

\section{Gaia and Planetary Nebulae}

Gaia will detect the central stars of many planetary nebulae down to a G magnitude of 20.7. The only parallaxes available in Gaia DR1 are those of the TGAS for stars brighter than a visual magnitude of $\sim 11.5$ (Lindegren et al. 2016). Thus only the brightest PN are likely to have observable parallaxes from the Gaia DR1 release. This indeed is the case, with only a small number $(<20)$ of the brightest central stars (Stanghellini et al. 2016) with a measured Gaia DR1 parallax. However, the situation will greatly improve with much more extensive set of parallaxes available with upcoming Gaia data releases, from Gaia DR2 onwards†.

Gaia is optimised for the detection of point sources, and in general is not sensitive to extended objects (with sizes $>0.5$ arcsec) (de Bruijne et al. 2015). The left side of Fig. 1 shows part of a Gaia Sky Mapper (SM) CCD image overlaid with detected sources. Red markers are for the brightest stars $(\mathrm{G}<13 \mathrm{mag})$ which are downlinked as small images. Yellow markers are for stars $13<\mathrm{G}<16$ mag; these are binned in across-scan direction. The cyan markers are the faintest stars with $16<\mathrm{G}<20$ mag where across-scan binning is done as well. Square shape markers, indicate those stars that will be used for Gaia attitude control.

However, as noted in de Bruijne et al. (2015), Gaia is able to resolve point like and somewhat extended structure. The exact performance of Gaia in detecting non-point like objects is complex, and is a function of object morphology, brightness and at which scan angle Gaia observes the source. In this respect, many features will only be observed at certain Gaia scan angles.

The sub-structure within extended objects such as PN is thus potentially detectable as point-like sources by Gaia. Sampling of these points may enable a detailed view of extended structure within the nebula. The capability of Gaia in this area was demonstrated by the early Ecliptic Pole Scanning observations of the large PN NGC 6543‡, where the complex nebula is decomposed by Gaia into thousands of individual mapping points. The right hand image of Fig. 1 shows the $\sim 84000$ Gaia detections that were made in the area around NGC 6543 from 25 July to 21 August 2014.

$\dagger$ The Gaia data release scenario is available at http://www.cosmos.esa.int/web/gaia/release

$\ddagger$ The Gaia Image of the Week (IoW_20141205) explores this in more detail, see http://www. cosmos.esa.int/web/gaia/iow_20141205 

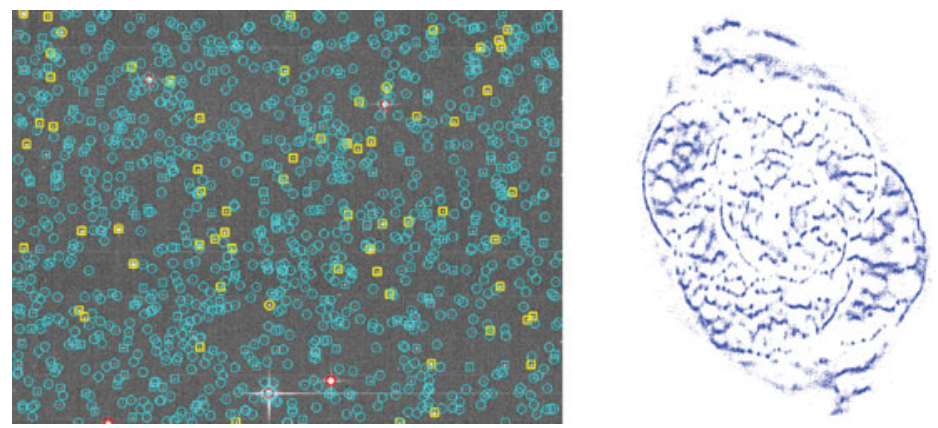

Figure 1. The left hand image shows a Gaia Sky Mapper image of a field obtained during commissioning (Image: ESA/Gaia/DPAC/Airbus DS). The right hand image shows the $\sim 84,000$ Gaia detections that were made in the area around NGC 6543 from 25 July to 21 August 2014 (Gaia image credit: ESA/Gaia/DPAC/UB/IEEC)

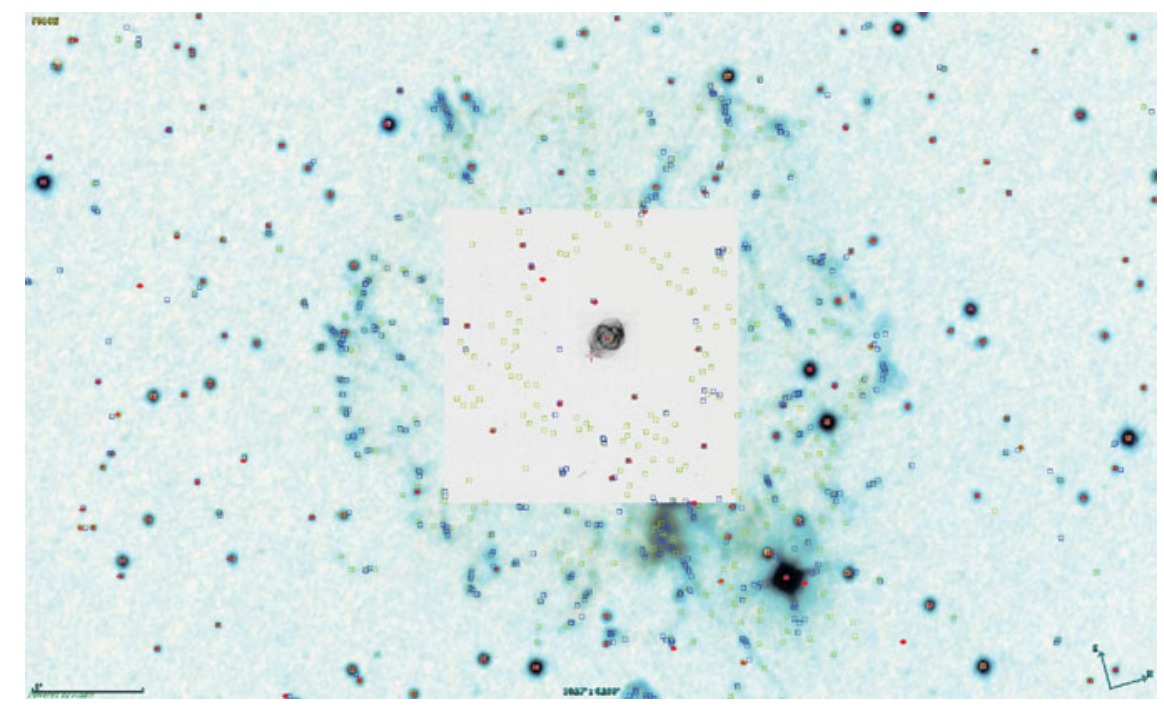

Figure 2. NGC 6543 as seen in Gaia DR1. The red dots are Gaia DR1 source detections, whilst the blue dots are USNO B1.0, and green dots GSC 2.3 sources. The background image is from DSS, whilst the central region is HST, both taken from CDS/Aladin.

With the five year nominal lifetime Gaia astrometry, it should be possible to accurately map the evolution of the plane of sky expansion of PN such as NGC 6543 and from line of sight velocities, the complex dynamical structure of the expanding nebula.

\section{The Morphologies of Planetary Nebulae as Revealed by Gaia}

Many PN have complex structure as described in a number of talks elsewhere in these proceedings. The PN morphologies can help infer the presence of, for instance, binary central stars.

Initial searches of the Gaia DR1 catalogues were made around a number of the brighter PN. One example is searching for source detections around NGC 6543. Fig. 2 shows the DSS image around NGC 6543, together with a high resolution HST image of the central region. Overlaid are the detections of stars as given in the USNO B1.0 and GSC 2.3 catalogues, along with detections in the Gaia DR1 catalogue. It is immediately apparent 
that there are very few Gaia source detections in and around NGC 6543. The question is where have the many thousands of detections that were indicated after commissioning gone?

The Gaia DR1 release paper (Gaia Collaboration et al. 2016a) notes that, as part of the data validation processes, there was a significant filtering of sources detected by Gaia but not published in the release. Filtering occurred at the processing sub-system level, thus for astrometry (Lindegren et al. 2016), for photometry (van Leeuwen et al. 2016), and at the global level (Arenou et al. 2016) where all data was combined for the data release. It is a combination of several of these filters that has resulted in the low number of detections in the nebula in this first data release. For instance, the requirement that each source should have been observed by Gaia at least 5 times meeting a threshold uncertainty limit.

The NGC 6543 observations were obtained in the early operational stages of Gaia with the at launch video processing unit (VPU) parameters active. As noted in de Bruijne et al. (2015), these govern the detection of sources in the SM Gaia CCDs. The detection algorithm automatically uses full-frame SM data to discriminate stars from spurious objects, such as cosmic rays and solar protons, using PSF-based criteria; the parameter settings adopted at launch ensured a high level of completeness down to the faint limit at $\mathrm{G}=20.7 \mathrm{mag}$, but with spurious detections in the (diffraction) wings of bright stars essentially passing unfiltered. In May 2016, a new set of parameters was uploaded that accepts fewer false detections at the expense of a reduced detection efficiency of objects beyond 20 mag. It is anticipated that these VPU parameters will be used for the remainder of the mission.

The new detection parameters will reduce the number of faint extended sources detected by Gaia. However, with the increasing number of scans and thus Gaia data of PN, it is fully expected that sufficient detections will be available of sub-structure within the nebula of PN such as NGC 6543 to ensure highly precise measures of PN morphologies to be undertaken.

\section{Acknowledgements}

This work has made use of data from the European Space Agency (ESA) mission Gaia (http://www.cosmos.esa.int/gaia), processed by the Gaia Data Processing and Analysis Consortium (DPAC, http://www.cosmos.esa.int/web/gaia/dpac/consortium). Funding for the DPAC has been provided by national institutions, in particular the institutions participating in the Gaia Multilateral Agreement.

The Digitized Sky Surveys were produced at the Space Telescope Science Institute under U.S. Government grant NAG W-2166.

This research has made use of "Aladin sky atlas" developed at CDS, Strasbourg Observatory, France.

\section{References}

Arenou, F., Luri, X., Babusiaux, C., et al. 2016, A\&\&A (special Gaia issue) J. H. J. de Bruijne, J. H. J., Allen, M., Azaz, S., et al. 2015, A\&\&A, 576, A74 Evans, D. W., Riello, M., De Angeli, F., et al. 2016, A\&A (special Gaia issue) Gaia Collaboration, Brown, A. G. A., Vallenari, A., et al. 2016, A\&SA, 595, A1 Gaia Collaboration, Prusti, T., de Bruijne, J. H. J., et al. 2016, A\&AA, 595, A1 van Leeuwen, F., Evans, D. W., De Angeli, F., et al. 2016, A\&A (arXiv:1612.02952) Lindegren, L., Lammers, U., Bastian, U., et al. 2016, A\&AA, 595, A4

Michalik, D., Lindegren, L., \& Hobbs, D. 2015 A $\& A$, 574, A115

Stanghellini, L., Bucciarelli, B., Lattanzi, M. G., \& Morbidelli, R. 2016 (arXiv:1609.08840) 